\title{
Experimental determination of the emissivity of bone
}

\author{
Arne Feldmann, Philippe Zysset* \\ Institute for Surgical Technology and Biomechanics, Stauffacherstrasse 78, University of \\ Bern, Switzerland
}

\begin{abstract}
Cutting and drilling operations in bone are involved in many orthopedic and otolaryngological surgeries. The temperature elevation of these procedures is potentially harmful to bone and soft tissue cells. The research on this topic aims therefore at minimizing temperature elevation and finding optimal process parameters. Experimental studies are mostly carried out on ex vivo setups using bovine bone material. For temperature measurements, either thermocouples or infrared cameras are used. Infrared cameras have potential advantages, but the emissivity value of the material has to be known. Literature values are scattered and vary within a wide range. An experimental study was carried out to quantify the emissivity using freshly frozen bovine and human bone, as well as human bone samples which were either fixed with Formalin or Thiel solution. Additionally, different surface finishes were used and emissivity was evaluated at different temperatures. The mean emissivity of bone was determined to be $\varepsilon=0.96 \pm 0.01$ for temperature elevations up to $60^{\circ} \mathrm{C}$. A slightly higher value of $\varepsilon=0.97 \pm 0.01$ was found for temperatures around $80^{\circ} \mathrm{C}$. No significant differences for human or bovine bone samples, preparation or fixation techniques were found.
\end{abstract}

Wordcount: 2380 words

Keywords: Emissivity constant, thermal camera, human bone, bovine bone, bone drilling, formalin and thiel fixation

${ }^{*}$ Corresponding author: philippe.zysset@istb.unibe.ch

Preprint submitted to Elsevier

April 13, 2016

(C) 2016. This manuscript version is made available under the Elsevier user license http://www.elsevier.com/open-access/userlicense/1.0/ 


\section{Introduction}

Within the last decade, the research on bone drilling and sawing has regained a lot of attention. The research focuses on modelling and experimentation of cutting forces and temperature elevations 1] [2]. Especially the produced heat is of concern, because high temperatures can lead to bone necrosis $\left(47^{\circ} \mathrm{C}\right.$ for 1 minute [3]) or can harm nerves and other structures for example during head and neck (otolaryngological) surgeries [4]. Even though this topic has gained interest, no final conclusions for example on the most effective drilling parameters for reducing temperature elevation have been drawn and the research is ongoing.

In general, there are two ways to measure the heat elevation in bone: the contact and non-contact measurements. For the contact measurement, the temperature is measured with thermocouples, which are inserted into the bone close to the drilling channel. The disadvantages of contact measurements concern the contact of thermocouple and bone (normally using a thermal paste), the difficulties of precise placement and the limitation to point temperature measurements. The non-contact temperature measurements make use of pyrometers or infrared cameras and are able to read out a temperature field. However, just the surface temperature can be measured. Additionally, it is necessary to know the emissivity value of the measured material to get the correct temperature reading. The emissivity $[\varepsilon]$ of a material is a property which provides a measure of how efficiently a surface emits energy relative to a black body. The maximum value is therefore $\varepsilon=1$ and cannot be reached by any material. The emissivity is often called a constant, but it can vary with different factors (infrared wavelength, temperature, surface roughness, viewing angle and sample geometry) [5]. These factors are discussed later, but with some assumptions it is possible to reduce their influence. For example, when the assumption is made that a material's emissivity does not dependent on wavelength, it is called a gray body emitter. If a constant can be defined, it is called the total emissivity of a material.

In the recent literature, when an infrared camera is used for bone temperature 
measurement, the bone surface is either covered with a carbon spray to increase the emissivity constant, or an emissivity value is assumed. The disadvantage of using a carbon, graphite or chalk spray as done by many authors [6] [7] [8] [9] is that the exact emissivity is not known (ca. $\varepsilon=0.95$ ) and the temperature fluctuation might be changed by this additional layer.

There have been some efforts to define the emissivity of bone. Stumme et al. 10. measured it to be $\varepsilon=1.01$, but this value is physically invalid. Other studies defined the constant, without explaining the procedure, to be 0.99 [11] [12]. This is very unlikely because it is nearly the emissivity of a black body. To the knowledge of the author, there is no material with an emissivity of $\varepsilon=0.99$. Another study [13] defined it to be $\varepsilon=0.41$ which is a very low value because in general non-metal materials have a high emissivity of $\varepsilon>0.9$ [5]. These quoted emissivity values highlight the need for a reliable value which can be used for future research.

\section{Material and Methods}

Before the beginning of the experiment, 18 samples (3 samples each; dimensions: ca. $20 \mathrm{~mm} \times(10 \mathrm{~mm}$ to $30 \mathrm{~mm}) \times 2 \mathrm{~mm})$ were prepared. Table 1 shows an overview of the different samples, which were all cut from the cortical bone layer in the longitudinal direction. Bovine bone samples, from the tibiae of four year old cows, were acquired from a local slaughterhouse and freshly frozen. Human samples (femur of 105 year old female) were either freshly frozen or were fixed with Formalin or Thiel fixation for more than 6 months. The distinction of cut and no-cut means that the top surfaces of samples were either cut with a diamond band saw (Exakt, Germany) or the outer bone surface was left blank (tissues and periosteum were removed). Additionally, 3 samples were cut with a hand saw to create a rough surface. The aim of the different surface finishes is to evaluate if there is an influence on the emissivity because of the variety of experimental setups and preparation methods reported in literature. 
Table 1: Different sample types, preparation and cutting techniques used for the evaluation of the emissivity value. Three samples were prepared for each group.

\begin{tabular}{l|l|l|l} 
No. & Bone & Preparation & Cutting \\
\hline $\mathbf{1}$ & Human & Fresh frozen & cut \\
\hline $\mathbf{2}$ & Human & Fresh frozen & no cut \\
\hline $\mathbf{3}$ & Human & Thiel Fixation & cut \\
\hline $\mathbf{4}$ & Human & Formalin Fixation & cut \\
\hline $\mathbf{5}$ & Bovine & Fresh frozen & cut \\
\hline $\mathbf{6}$ & Bovine & Fresh frozen & no cut
\end{tabular}

For each individual experiment, three samples from different groups were attached onto an aluminum plate. A thin layer of thermal conductive paste (MX-4, Arctic, Switzerland) was applied in-between to ensure an even heat distribution. A stripe of black electrical insulating tape (Super 33+, 3M, USA) with known emissivity $(0.96[14]$ ) was attached on the top surface of the bone samples so that half of the surface area is covered. The plate was then placed onto a laboratory heating plate and all other surfaces around the samples were covered with black chalk spray or the electrical tape to reduce reflections (apparent temperature).
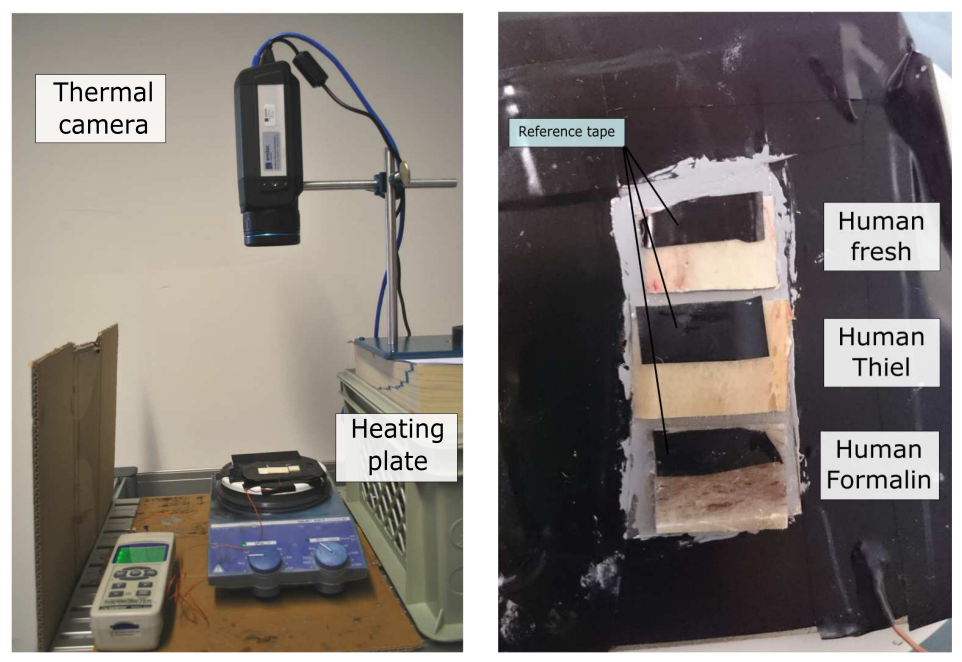

Figure 1: Left: Experimental setup with thermal camera and heating plate. A thermocouple was used for adjustment of heating plate temperature; Right: Example of three bone samples with attached reference tape. 
A thermal camera (FLIR IR 655sc, FLIR Systems, USA) was placed vertically over the samples in a focus distance of $0.3 \mathrm{~m}$ (see Figure 1). The plate was heated up step wise to $40^{\circ} \mathrm{C}, 60^{\circ} \mathrm{C}$ and $80^{\circ} \mathrm{C}$, because the emissivity should be measured 20 Kelvin above room temperature [14]. The black insulation tape was used as a reference because of the known emissivity constant. At least 5 minutes were waited after heating the plate to ensure temperature equilibrium within the samples. This time period has been found sufficient in pilot tests. By then, it was assumed that the tape and the bone surface had the same evenly distributed temperatures. For temperature readouts, average values were used from four defined areas (ca. $5 \mathrm{~mm}$ x $5 \mathrm{~mm}$ ) on evenly heated parts (see Figure 2) of bone surface and tape. For each temperature elevation, the emissivity of each bone sample was then found by changing the emissivity value in the software (FLIR Research R\&D) so that bone surface temperature equaled the reference temperature.

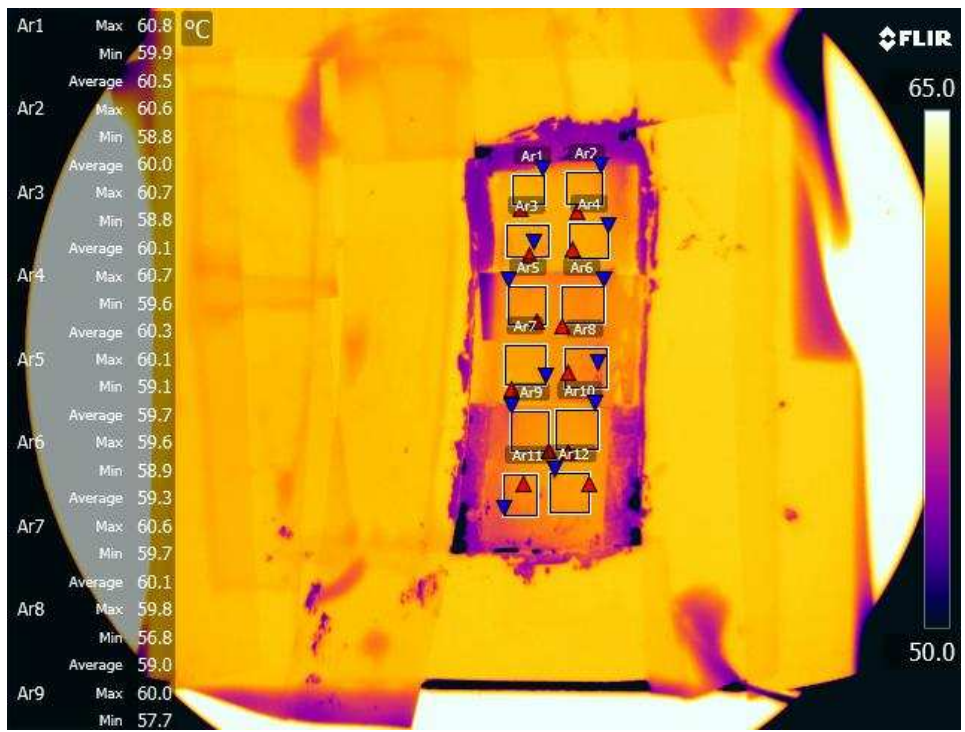

Figure 2: Picture from the thermal camera showing the three samples from Figure 1 at $60^{\circ} \mathrm{C}$. The average temperatures in two rectangular areas of the reference tape and bone was used to find the emissivity value for each sample. 


\section{Results}

An analysis of covariance (ANCOVA) was carried out using temperature as a continuous and sample type as categorical explanatory variable. No conclusive differences between the different bones, preparation techniques or cutting methods were found. A small but significant trend towards higher emissivity with higher temperatures was found $\left(R^{2}=0.231, p<0.001\right)$. The emissivity values were then grouped for each temperature elevation as shown in Figure 3. A mean value of $\varepsilon=0.96 \pm 0.01$ was found for temperature elevations of $40^{\circ} \mathrm{C}$ and $60^{\circ} \mathrm{C}$. An increase to $\varepsilon=0.97 \pm 0.01$ can be seen for a temperature raise to $80^{\circ} \mathrm{C}$. A pairwise wilcoxon rank-sum test revealed $p<0.05$ for the comparison of grouped data of $40^{\circ} \mathrm{C}$ and $60^{\circ} \mathrm{C}$ as well as $60^{\circ} \mathrm{C}$ and $80^{\circ} \mathrm{C}$. A more significant value $(p<0.001)$ was found when comparing $40^{\circ} \mathrm{C}$ and $80^{\circ} \mathrm{C}$.

\section{The emissivity constant of bone}

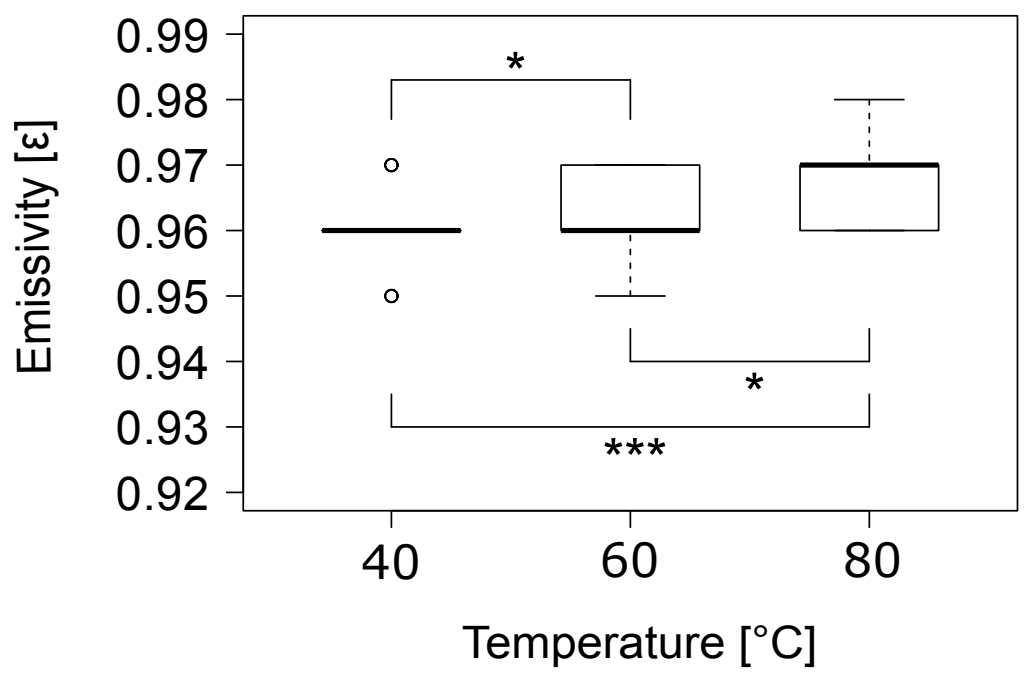

Figure 3: Grouped emissivity values for each temperature elevation. A mean emissivity value of $\varepsilon=0.96 \pm 0.01$ was found for $40^{\circ} \mathrm{C}$ and $60^{\circ} \mathrm{C}$, while the value increased to $\varepsilon=0.97 \pm 0.01$ for $80^{\circ} \mathrm{C}$. Pairwise wilcoxon rank-sum test results are shown as well $(*: p<0.05 ; * *: p<$ $0.01 ; * * *: p<0.001)$ 


\section{Discussion}

As mentioned before, the emissivity of a material can change with infrared wavelength, temperature, surface roughness, viewing angle and sample geometry. Not all of these factors influence all kinds of materials, so that the wavelength dependency is especially important for metals while other materials can be defined as a gray body. For this experiment, the infrared wavelength of the thermal camera was used $(7.5-14 \mu m)$. Additionally, the FLIR R\&D program corrected all temperature values with the input values of humidity, room temperature and reflected environment radiation (apparent temperature). The sample geometry (thickness) is important for very thin or non-opaque objects because they can have a so called shine-through effect. With a thickness of 2 $\mathrm{mm}$ the samples have a sufficient thickness. The viewing angle is said to be not an influencing factor as long as the viewing angle is not lower than $40^{\circ}-45^{\circ}$ and the sample has therefore a so called diffuse, gray surface.

The used procedure to determine the emissivity constant follows the FLIR reference handbook [14], but should be understood as an approximation of the real value. Difficulties with this procedure include the unknown uncertainty the emissivity value of the tape, the equal distribution of temperature within the samples, the assumption of equal temperature of bone surface and tape and the manual process in finding the emissivity constant with the software.

To reduce some of these possible errors, an average temperature was used for finding the emissivity in an area which was placed on an apparent equally distributed part of the bone and tape. Additionally, three different samples were used for each experiment. As mentioned, no significant difference between human or bovine bone was found and the differences between fresh, fixation and cutting techniques are within the measurement errors. A small dependency $\left(R^{2}=0.231\right)$ of temperature on the emissivity value was found. Further validation of the suggested emissivity using a larger temperature range is necessary and should be facilitated in a more controlled environment (e.g. heating chamber). 
The found mean emissivity value of $\varepsilon=0.96-0.97$ lies in-between the emissivities of plants and animals $\varepsilon \approx 0.95$ and human skin $\varepsilon=0.98$ [15] and coincides with the general high emissivities of biological tissues. Hence no difference was observed for the other parameters, it is valid for bovine and human bone (and most likely for other species as well). Nevertheless we only investigated mature bones (105 year old human and 4 year old cow) so that we cannot rule out different values for younger bones. The found value should function as a first approximation and can be modified to account for different experimental environments. It should be noted, that a change in the emissivity constant of 0.01 results in a change of read temperature in $0.1-0.5^{\circ} \mathrm{C}$ depending on the bone temperature (higher temp $\rightarrow$ higher error).

\section{Conclusion}

Summarizing all the measurements, the authors recommend using an average emissivity value of $\varepsilon=0.96 \pm 0.01$ for temperatures up to $60^{\circ} \mathrm{C}$. A higher value of $\varepsilon=0.97 \pm 0.01$ should be used for temperatures around $80^{\circ} \mathrm{C}$. This temperature trend needs to be confirmed by experiments using a wider temperature range. A variety of parameters were tested and no significant differences between human, bovine, fixation (formalin, thiel and fresh) and cutting technique were found. Over all, it was possible to define the emissivity value for bone which can be used for future research.

\section{Acknowledgment}

The authors would also like to thank Nano Tera and the Swiss National Science Foundation for funding the research within the hear restore project (RTD 2013) as well as Susanne Boemke of the Anatomy Institute of the University of Bern for providing the bone samples. These ex vivo human bone samples were acquired and used according to the ethics rules and approval of the Anatomy Institute (University of Bern, Switzerland). 
REFERENCES

\section{References}

\section{References}

[1] M. Marco, M. Rodriguez-Millan, C. Santiuste, E. Giner, M. H. Miguelez, A review on recent advances in numerical modelling of bone cutting, Journal of the Mechanical Behavior of Biomedical Materials 44 (2015) 179-201. doi:10.1016/j.jmbbm.2014.12.006

[2] J. Lee, O. B. Ozdoganlar, Y. Rabin, An experimental investigation on thermal exposure during bone drilling, Medical engineering \& physics 34 (10) (2012) 1510-20. doi:10.1016/j.medengphy.2012.03.002

[3] R. Eriksson, T. Albrektsson, The Effect of heat on bone regeneration: An experimental study in the rabbit using the bone growth chamber, Journal of Oral and Maxillofacial Surgery 42 (1984) 705-711.

[4] R. F. Labadie, R. Balachandran, J. H. Noble, G. S. Blachon, J. E. Mitchell, F. a. Reda, B. M. Dawant, J. M. Fitzpatrick, Minimally invasive imageguided cochlear implantation surgery: First report of clinical implementation, The Laryngoscope (2013) 1-8doi:10.1002/lary.24520

[5] M. Kaviany, Principles of Heat Transfer, Wiley-Interscience, Michigan, 2001.

[6] T. Udiljak, D. Ciglar, Investigation into bone drilling and thermal bone necrosis, Advanced in Production Engineering \& Management 2 (2007) $103-112$.

[7] G. Augustin, S. Davila, T. Udiljak, D. S. Vedrina, D. Bagatin, Determination of spatial distribution of increase in bone temperature during drilling by infrared thermography: preliminary report., Archives of orthopaedic and trauma surgery 129 (5) (2009) 703-9. doi:10.1007/s00402-008-0630-x.

[8] J. Soriano, P. J. Arrazola, Effects of rotational speed and feed rate on temperature rise, feed force and cutting torque when drilling bovine cortical bone 408 (2012) 408-416. doi:10.1063/1.4707590 
[9] J. Soriano, P. J. Arrazola, Effects of Rotational Speed, Feed Rate and Tool Type on Temperatures and Cutting Forces When Drilling Bovine Cortical Bone, Machining Science and Technology 17 (4) (2013) 611-636. doi:10.1080/10910344.2013.837353

[10] L. D. Stumme, T. H. Baldini, A. Jonassen, J. M. Bach, M. Golden, C. Health, EMISSIVITY OF BONE (1).

[11] R. Baker, M. Whitehouse, M. Kilshaw, M. Pabbruwe, R. Spencer, A. Blom, G. Bannister, Maximum temperatures of $89^{\circ} \mathrm{C}$ recorded during the mechanical preparation of 35 femoral heads for resurfacing., Acta orthopaedica 82 (6) (2011) 669-73. doi:10.3109/17453674.2011.636681

[12] E. B. Dolan, T. J. Vaughan, G. L. Niebur, C. Casey, D. Tallon, L. McNamara, How bone tissue and cells experience elevated temperatures during Orthopaedic Cutting: An Experimental and Computational Investigation., Journal of biomechanical engineering (c). doi:10.1115/1.4026177.

[13] M. A. L. Freire, J. C. R. González, E. S. Tapia, XIII Mediterranean Conference on Medical and Biological Engineering and Computing 201341 (2014) 97-100. doi:10.1007/978-3-319-00846-2.

[14] G. Orlove, FLIR - R\&D manual/ ITC Technical publication 29 (2011).

[15] C. Villaseñor Mora, An indirect skin emissivity measurement in the infrared thermal range through reflection of a $\mathrm{CO} 2$ laser beam, Revista mexicana de ...55 (5) (2009) 387-392. 